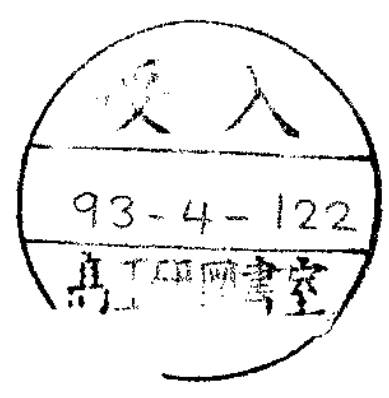

EUROPEAN ORGANIZATION FOR NUCLEAR RESEARCH

CERN-PPE/93-04

January 6, 1993

\title{
Performance of Gas Microstrip Chambers on Glass Substrates with Electronic Conductivity
}

\author{
R.Bouclier, G.Million, L.Ropelewski and F.Sauli \\ (CERN, Geneva, Switzerland)
}

Yu.N.Pestov and L.I.Shekhtman

(BINP Novosibirsk, Russia)

\begin{abstract}
We present some recent measurements on Gas Microstrip Chambers (GMSC) realised on glass substrata with different bulk resistivity. Chambers manufactured on low resistivity supports $\left(\sim 10^{9} \mathrm{Ohm} \cdot \mathrm{cm}\right)$ have very high rate capability, up to and above $5 \cdot 10^{6}$ counts $\mathrm{mm}^{-2} \cdot \mathrm{s}^{-1}$ at proportional gains around $10^{3}$. With increase of resistivity of the support, the rate capability decreases, presumably because of the modification of electric field induced by the current flowing along the resistive surface, and at $\sim 10^{12} \mathrm{Ohm} \cdot \mathrm{cm}$ the gain is affected already at the level of $\sim 5 \cdot 10^{5}$ counts $\mathrm{mm}^{-2} \cdot \mathrm{s}^{-1}$. Long term measurements of gain show a stable behaviour for the chambers built on low resistivity glass, up to an integral charge of $\sim 130 \mathrm{mC}$ per $\mathrm{cm}$ of strip. At normal operating conditions (an avalanche size of about $10^{5}$ electrons) this corresponds to $10 \mathrm{Mrad}$ of absorbed dose for minimum ionizing particles, thus making the GMSC a good candidate for the use in LHC and SSC detectors. In order to reach the quoted lifetime, particular precautions have to be taken to guarantee gas purity.

The present study has been realised in the framework of the collaboration for the Development of GMSC for Radiation Detection and Tracking at High Rates (CERN Research and Development Project RD28).
\end{abstract}




\section{Introduction.}

The recently developed Gas Microstrip Chambers (GMSC) [1] have very promising features, making the devices intrinsically capable to overcome some shortcomings of existing wire chambers and namely limited spatial resolution and rate capability. Significant improvements have been achieved through a reduction of the spacing between amplifying electrodes, obtained using precise microelectronics technology, photo- or electron-beam lithography to produce metal strip structures on top of insulating substrata (Fig. 1).

Results reported by various groups developing GMSCs are very encouraging: a position accuracy for minimum ionising particles of around $30 \mu \mathrm{m}$ rms, better than what can be achieved with drift chambers and close to the one of silicon microstrip detectors [2]; a rate capability higher than in any other type of proportional gas chamber, up and above $10^{6} \mathrm{~mm}^{-2} \mathrm{~s}^{-1}$ [3]; an energy resolution of $10.7 \%$ fwhm for $\mathrm{Fe}^{55} 5.9 \mathrm{keV} \mathrm{x}$-rays, achieved at proportional gains up to $10^{4}$ [4]. These properties make GMSCs a very attractive choice to implement an intermediate tracking detector for the experimental set-ups designed for the future high luminosity colliders LHC and SSC [5-7].

Recent observations have shown however certain instabilities in the performance of GMSC. Chambers produced on high resistivity substrata, such as Hoya Soda Lime (SL), Pyrex or Tempax glass, and various plastic foils exhibit modifications of gain in measurements at relatively moderate or high fluxes $[3,8$, 9]. A systematic study on these phenomena shows that they can be divided into short-term, reversible and reproducible changes of gain, and long-term modifications which, in general, lead to permanent modification of signals in the irradiated region. The degradation of gain is often accompanied by visible changes in the microstrip structure, appearing as deposits on the surface and/or damages of the strips.

Short-term effects, causing a relatively fast (seconds or minutes) drop of gain after the beginning of operation, can in turn be of two types, global and local phenomena [3]. After turning high voltage on, the gain can decrease all over the surface of the chamber and this independently from the irradiation rate; the drop is usually measured by a short probing of the signal at the lowest possible radiation level. An additional gain drop can be observed in the exposed area: during the irradiation the gain decreases until reaching a plateau, the value of which is however rate dependent. This behaviour can be repeated many times in the same or different areas, and it is understood to be due to the accumulation on the insulating surface of a fraction of the ions produced in the avalanches. The effect can be reduced by a proper choice of the back plane potential, and avoided all together using a lower resistivity substratum [1,3,10-12].

Long-term, non-reversible changes of gain are due to a process similar to the polymerization or aging observed since long time in wire chambers exposed to high radiation levels. They appear to depend critically on the choice of the substratum material, metal for cathodes and anodes, working gas mixture and on the presence of pollutants both in the gas and on the surface of the structures which could be a consequence of the manufacturing process; it is a common finding that GMSC are particularly prone to this kind of irreversible degradation.

This paper presents the results of comparative tests of chambers manufactured on various semi-conducting and insulating supports, with the aim to find the optimal resistivity in order to allow high-flux stable operation. We also report some preliminary observations on the dependence of long-term gain modifications on the nature of the metal used for the strips, and on the manufacturing technique. 


\section{Experimental set-up and chambers construction}

For the measurements here described we have used several chambers built on glass substrata with bulk resistivity in the range from $10^{9}$ to $10^{15} \mathrm{Ohm} \cdot \mathrm{cm}$. The lower resistivity glasses have electronic conductivity, and were specially developed and produced for our needs $[13,14]$. Two chambers were manufactured on special electronic conductive glass plates having a bulk resistivity of $10^{9}$ and $10^{12} \mathrm{Ohm} . \mathrm{cm}$ [15]; they are referred to as MS1 and MS2, respectively (see Table 1). The strip structure was produced by electron beam lithography on a vacuum evaporated titanium adherence under-layer. A gold layer, galvanically grown on the titanium under-layer, was then etched by a combination of wet and plasma processing. Another chamber, MS3, used for comparative tests, was also manufactured on a glass substrate with bulk electronic conductivity of about $10^{12}$ Ohm.cm, using a conventional wet etching technique of a chromium layer [16]. All chambers had similar geometry, with anode and cathode strips alternating on the substratum at a distance of $100 \mu \mathrm{m}$ between centers and with a width of 10 and $80 \mu \mathrm{m}$ respectively. A schematic cross section of the chambers was shown in Fig. 1.

In order to verify the consistency of these measurements with earlier results, as well as a reference for comparisons with the chambers made on lower resistivity substrata, we have measured also the performance of a GMSC manufactured on a standard Hoya SL glass identical to the one described in Ref. 3 (MS4, see table 1); the bulk resistivity of this glass is $10^{15} \mathrm{Ohm} \cdot \mathrm{cm}$, increasing by at least one order of magnitude after application of the potentials presumably because of an ion conduction mechanism. This chamber has a wider pitch, 200 $\mu \mathrm{m}$ between centers of anode and cathode strips, 10 and $50 \mu \mathrm{m}$ wide respectively and made of chromium.

The experimental set-up used for the measurements is shown schematically in Fig. 2. Individual GMSC plates are installed in a stainless steel box with a front aluminium window $50 \mu \mathrm{m}$ thick and metal-glass feed-through allowing the application of operating potentials and extraction of signals. We avoided as much as possible the use of any organic material in the construction, in order not to affect long-term tests. The chamber plate is mounted with insulating rods inside the box, with connections for providing the back plane and anode potentials; the drift electrode is realized with a stainless steel mesh mounted at 10 $\mathrm{mm}$ from the surface of the plate itself. All anodes of the chambers are connected together and to a high voltage power supply through an RC noise filter, as shown schematically in Fig. 2; cathode strips are connected together in 6 groups 25 strips each, grounded through a $100 \mathrm{kOhm}$ resistor in order to allow the recording either of pulse height with the help of charge sensitive amplifier coupled to a shaping amplifier and a fast charge ADC, or of the current deduced from the measurements of the voltage drop on the resistor using a sampling ADC. At low radiation fluxes, both pulse height on individual counts and current could be measured, while to investigate the performance at high fluxes (above $10^{4} \mathrm{~mm}^{-2} \mathrm{~s}^{-1}$ ) we have measured only the current through the chamber thus avoiding pile-up problems.

Electrical connections to the electrodes on the chamber's plate are made with minute drops of two-components conductive epoxy; to prevent discharges at the edges of the anode and cathode strips, the ends of the plates are covered with a thin layer of standard epoxy. Particular care is taken in the curing of the epoxy, to avoid as much as possible outgassing that could pollute the chamber. All the measurements were performed with an argon-dimethyl ether gas mixture (90-10) at atmospheric pressure; we used a custom built clean gas system, with all tubing made of stainless steel and mass flow meters containing no organic materials in order to avoid pollution during long-term aging tests. The test box is mounted in 
making a practical use of the device in a non-uniform radiation flux rather problematic.

In the chambers with a low resistivity support having electronic conductivity these instabilities become smaller. The chamber made on the $10^{12}$ $\mathrm{Ohm} \cdot \mathrm{cm}$ glass substratum (MS2) still shows however a gain drop at very high rates. This is seen in Fig. 6, where the measured gain is provided for MS2 as a function of time after the beginning of irradiation, and at increasing fluxes. In Fig. 7 one can see the comparison of the gain variation in time of MS1 and MS2 irradiated with a flux of $10^{6} \mathrm{~mm}^{-2} \mathrm{~s}^{-1}$; for the lower resistivity support, the gain is essentially constant within measurement errors.

\subsection{Rate capability}

The described fast modifications of gain in GMSCs made on high resistivity support affect the rate capability of the chamber. This can be seen in Fig. 8, showing the gain as a function of rate for MS1 and MS2; measurements were realized with an increasing flux of $8 \mathrm{keV} \mathrm{x}$-rays, at an avalanche size of about $3 \cdot 10^{5}$ electrons. One can attribute the gain drop at high rates observed with the low resistivity device MS1 to space charge phenomena in the gas (accumulation of positive ions affecting the gain); the decrease at lower rates observed on MS2 is obviously due to accumulation of charges on the surface of the support, not sufficiently conducting (all other manufacturing and operating parameters in the two chambers being identical). Note that even for the higher resistivity device, the rate capability of above $10^{5} \mathrm{~mm}^{-2} \mathrm{~s}^{-1}$ exceeds the requirements for an LHC or SSC intermediate tracking detector.

\subsection{Long-term stability of operation}

We have studied the long-term stability measuring variations of gain of the chambers constantly exposed with power on to high radiation fluxes over several weeks. The measured parameter is in this case the DC current through the chamber, sampled at regular intervals; at the same time we have monitored small variations in the flux and gas conditions using the single wire proportional counter as described in the section 2 . The dependence of relative gain on accumulated charge, normalized to a $\mathrm{cm}$ length of strip, for MS1 and MS2 is shown in Fig. 9. The duration of the measurement was about 10 days in both cases; with MS1 the radiation rate was $2 \cdot 10^{6} \mathrm{~mm}^{-2} \mathrm{~s}^{-1}$, while for MS2 we used a smaller rate $\left(5 \cdot 10^{5} \mathrm{~mm}^{-2} \mathrm{~s}^{-1}\right)$ in order to operate still in the region where the gain is not affected by the rate (see Fig. 8). After correcting for small gain variations due to ambient conditions and gas changes, using the monitor data, one can see that performance of MS1 is stable within the measurement errors up to 130 $\mathrm{mC} / \mathrm{cm}$ and of MS2 at least up to $30 \mathrm{mC} / \mathrm{cm}$. Assuming an avalanche size of $10^{5}$ electrons in the detection of charged particles, the integral charge withstood in MS1 corresponds to an exposure to about $10 \mathrm{Mrad}$ of minimum ionizing radiation.

We have also compared the long-term performance of two chambers manufactured with different technology, as described in section 2 , on similar electronic conductive substrata, MS2 and MS3. In Fig. 10 we can see that the behaviour of the two chambers is very similar, until a sudden jump of current was detected on MS3 after about $14 \mathrm{mC} / \mathrm{cm}$. Visual inspection of this chamber shows a permanent damage of the metal strips in the irradiated region, while MS2 worked without any problem up to $30 \mathrm{mC} / \mathrm{cm}$ and the surface of the structure did not show any visible degradation. $\mathrm{A}$ similar behaviour has been observed in other regions of the chambers; we do not know at this point if the difference in behaviour can be attributed to the metal used for the strips or to the manufacturing 
front of a collimated $x$-ray tube having an adjustable current in order to vary the radiation flux; at a distance of around $10 \mathrm{~cm}$, the tube provides in this geometry a maximum detected flux of about $5 \cdot 10^{6}$ photons $\mathrm{mm}^{-2} \mathrm{~s}^{-1}$, with an energy spectrum centered around $8 \mathrm{keV}$. A single wire proportional counter, used to monitor gas, flux and ambient changes is installed using the output gas from the GMSC box, and is irradiated with an attenuated fraction of the $x$-ray flux from a second collimator on the tube.

A small on-line computer is used for recording the data both from the test GMSC and from the monitor proportional tube over extended times of exposure.

\section{Experimental measurements and discussion of the results}

\subsection{General performance, leakage currents, pulse height spectra}

Due to the low bulk resistivity of the substratum, chambers manufactured on semi-conducting glass have a DC leakage current under application of voltage to the electrodes. Table 1 provides the values of this current for MS1, MS2 and MS3, normalized to unit area; the current was measured on groups of 25 cathodes connected to ground as described through a $100 \mathrm{kOhm}$ resistor, and with $300 \mathrm{~V}$ applied on the anode strips. We provide also in the table the equivalent surface resistivity of the structures, deduced from the measured current, in order to allow a comparison with chambers manufactured on materials with surface treatment. One should note that, despite the large value of the leakage current particularly for the lower resistivity substrata, the operation of the chamber is very stable and satisfactory. As shown in Fig. 3 (measured with MS1), the energy resolution for 8 $\mathrm{keV}$ is about $16 \%$ fwhm at a gain of $10^{3}$, a quite reasonable value; the leakage current on this chamber was close to $10 \mu \mathrm{A}$ on the group of 25 cathode strips, at the operating voltage. Similar energy spectra have been obtained with the higher resistivity supports, demonstrating that the noise contribution of the leakage current is in all cases rather small.

\subsection{Short-term modifications of gain}

As mentioned in the introduction, a change of gain after application of voltage (usually a decrease) has been observed on most microstrip structures manufactured on insulating supports, with a time constant that does not seem to depend on irradiation rates. However this kind of instability is absent in GMSC made on electronically conductive substrata, as can be seen in Fig. 4 where we compare the results obtained with MS2 and MS4. For this particular measurement, the chambers were exposed for about 10 seconds immediately after application of voltages to a localized low x-ray flux providing about 100 counts $\mathrm{mm}^{-2} \cdot \mathrm{s}^{-1}$ in order to allow a measurement of gain at time zero; the irradiation was then suspended, and resumed at regular intervals again only for the short time necessary to record the pulse height spectra (around 10 seconds). We call this procedure a no irradiation or zero rate measurement, although to record gain one has obviously to use some level of exposure. All structures tested by us and by other authors seem to reach a "stable" gain, more or less uniform over the surface, after minutes to hours from application of voltage.

After reaching a constant gain situation with the above mentioned no irradiation procedure, we have studied the rate-dependent gain modification on the chambers at increasing fluxes. In Fig. 5 the results of such measurements are shown for the chamber on high resistivity ionic glass (MS4). The irradiated area was about 3 by $3 \mathrm{~mm}^{2}$, and the drop of gain is verified to occur locally in the irradiated spot the rest of the chamber remaining unaffected. One can see that the measured gain reaches a plateau, whose value is however rate-dependent thus 
process. A more careful investigation is certainly required to clarify this aspect of the long-term operation.

\section{Conclusions and summary}

In a systematic comparison of behaviour of several GMSC detectors manufactured on glass substrata with electronic conductivity, we have confirmed the effect of support resistivity on rate capability, already suggested in previous works. Several distinct effects conjure to affect the operating stability of the device: a short term, rate-independent overall decrease of gain for the chambers made on ionic conductivity supports, with a time constant of minutes to hours, that can be tentatively understood as a polarization of the dielectric after application of the voltages. The amount and the time constant of the gain drop are large for the higher resistivity ionic supports $\left(10^{15} \mathrm{Ohm} . \mathrm{cm}\right)$, but are barely detectable for the lower resistivity electronic ones $\left(10^{9} \mathrm{Ohm} \cdot \mathrm{cm}\right)$.

Additional gain modifications are observed when the detectors are exposed to a high and steady flux of radiation. They appear to be due to two independent mechanisms: surface charging up and ageing. The first process is clearly dependent on the detected radiation flux and on the conductivity of the support; for the lower resistivity substrata tested, there is no gain drop observed up to a flux exceeding $10^{6}$ photons $\mathrm{mm}^{-2} \mathrm{~s}^{-1}$, and the drop observed above this rate is presumably due to space charge build-up in the gas. In clean gas and chamber conditions, and under continuous irradiation at a rate below this value, the gain remains stable up to a total charge of $130 \mathrm{mC}$ per $\mathrm{cm}$ of strip (equivalent to about $10 \mathrm{Mrad}$ of radiation dose); no visible change of the surface or damage of the metal strips is observed. Note that the situation was quite different when the same kind of long-term measurement were made on similar structures but using a more conventional standard (and probably polluted) gas mixing system and plastic tubing [3]; in that case, substantial evidence for permanent damage and thin film coating (aging) was obtained.

The best results in terms of long-term stability have been obtained with the chambers manufactured in gold by a combination of wet and plasma etching. We are investigating other structures, made by wet etching on chromium and gold, in order to ascertain the effect (if any) of the different electrode materials and fabrication technology.

\begin{tabular}{|c|c|c|c|c|c|}
\hline & Technology & $\begin{array}{c}\text { Pitch } \\
(\mu \mathrm{m})\end{array}$ & $\begin{array}{c}\text { Bulk Resistivity } \\
(\mathrm{Ohm} \cdot \mathrm{cm})\end{array}$ & $\begin{array}{c}\text { Leakage current } \\
\left(\mu \mathrm{A} / \mathrm{cm}^{2}\right) 300 \mathrm{~V}\end{array}$ & $\begin{array}{c}\text { Surface resistivity } \\
(\mathrm{Ohm} / \text { Square })\end{array}$ \\
\hline MS1 & $\begin{array}{c}\text { Gold, Wet and } \\
\text { Plasma Etching }\end{array}$ & 100 & $10^{9}$ & 10 & $9 \cdot 10^{11}$ \\
\hline MS2 & $\begin{array}{c}\text { Gold, Wet and } \\
\text { Plasma Etching }\end{array}$ & 100 & $10^{12}$ & 0.018 & $7 \cdot 10^{14}$ \\
\hline MS3 & $\begin{array}{c}\text { Chromium } \\
\text { Wet Etching }\end{array}$ & 100 & $2.10^{12}$ & 0.035 & $3 \cdot 10^{14}$ \\
\hline MS4 & $\begin{array}{c}\text { Chromium } \\
\text { Wet Etching }\end{array}$ & 200 & $>10^{15}$ & - & $>10^{17}$ \\
\hline
\end{tabular}

Table 1

Main construction parameters of the GMSC. Bulk resistivity is measured on glass plates before processing; surface resistivity is deduced from the leakage current measured between anode and cathode strips after manufacturing of the detector. 


\section{References}

1. A.Oed, Nucl. Instr. Methods A263 (1988) 351.

2. F.Angelini, R.Bellazzini, A.Brez, M.M.Massai, G.Spandre, M.Torquati, R.Bouclier, J.Gaudaen and F.Sauli, Nucl. Physics 23A (1991) 254.

3. R.Bouclier, J.J.Florent, J.Gaudaen, G.Million, A.Pasta, L.Ropelewski, F.Sauli and L.I.Shekhtman, Nucl. Instr. Methods in Phys. Res. A323 (1992) 236.

4. F.Angelini, R.Bellazzini, A.Brez, E.Focardi, T.Lomtadze, M.M.Massai, G.Spandre, M.R.Torquati, R.Bouclier, J.Gaudaen, F.Sauli and A.Perret, CERN 90-10 Vol. III p. 222 (Proc. LHC Workshop, Aachen 4-9 October 1990).

5. Solenoidal Detector Collaboration.Technical Report SDC-92-201 (April 1992).

6. CMS: The Compact Muon Solenoid. Letter of Intent CERN/LHCC 92-3 (1 October 1992).

7. ATLAS Letter of Intent, CERN/LHC 92-4 (10 October 1992)

8. J.E.Bateman, J.F.Connolly, R.Stephenson, Proc.European Workshop on x-Ray Detectors for Synch. Radiation, ed. A. Walenta (Aussois, September 1991) p.87.

9. R. Bouclier, J.J. Florent, J. Gaudaen, G. Million, L. Ropelewski and F. Sauli, IEEE Trans. Nucl. Sci. NS-39 (1992) 650.

10. J.J. Florent, J. Gaudaen, L. Ropelewski and F. Sauli, CERN-PPE/92-78 (1992). To be published in Nucl. Instr. Methods in Phys. Res.

11. F.Angelini, R.Bellazzini, L.Bosisio, A.Brez, M.M.Massai, A.Perret, G.Spandre and M.Torquati, Nucl. Instr. Methods in Phys. Res. A323 (1992) 229.

12.G.D.Minakov,Yu.N.Pestov,V.S.Prokopenko and L.I.Shekhtman, Performance of Gas Micro-Strip Chambers on Glass with Ionic and Electronic Conductivity, INP Novosibirsk 1992. Accepted for publ. by Nucl. Instr. Methods in Phys. Res.

13. SSPC NIIES, Moscow, Russia.

14. Stazione Sperimentale Vetro, Murano, Italy.

15. SRI "Vostok", Novosibirsk, Russia.

16. Baumer IMT, Greifensee, Switzerland.

\section{Figure captions:}

Fig. 1: Cross-section of a Gas Micro-Strip Chamber.

Fig. 2: Experimental set-up for the high rate, long term measurements.

Fig. 3: Pulse height spectrum for $8 \mathrm{keV}$-rays measured with the low-resistivity chamber MS1.

Fig. 4: Global modifications of gain, after application of voltage, on a low and high resistivity chambers (MS2 and MS4 respectively) in absence of irradiation (except for the time necessary for the measurement).

Fig. 5: Local modification of gain as a function of time in the region exposed to radiation, measured with the high resistivity ionic conductivity chamber MS4.

Fig. 6: Local gain modification with time at different rates, measured with MS2 (made on electronic conductivity glass with a resistivity of $\sim 10^{12} \mathrm{Ohm} \cdot \mathrm{cm}$ ).

Fig. 7: Local gain modification at very high flux measured with MS1 and MS2 ( $10^{9}$ and $10^{12} \mathrm{Ohm} \cdot \mathrm{cm}$ respectively).

Fig. 8: Gain as a function of rate measured with MS1 and MS2 at an avalanche size of $3 \cdot 10^{5}$ electrons.

Fig. 9: Long-term gain stability measured with MS1 and MS2. The avalanche size is the same for the two measurements $\left(2.4 \cdot 10^{5}\right.$ electrons) and a radiation flux of $2 \cdot 10^{6} \mathrm{~mm}^{-2} \mathrm{~s}^{-1}$ for MS1 and $5 \cdot 10^{5} \mathrm{~mm}^{-2} \mathrm{~s}^{-1}$ for MS2.

Fig. 10: Long-term gain stability measured with MS2 and MS3. In the last case, an irreversible increase of current with permanent damage was recorded after a total charge of around $14 \mathrm{mC}$. 


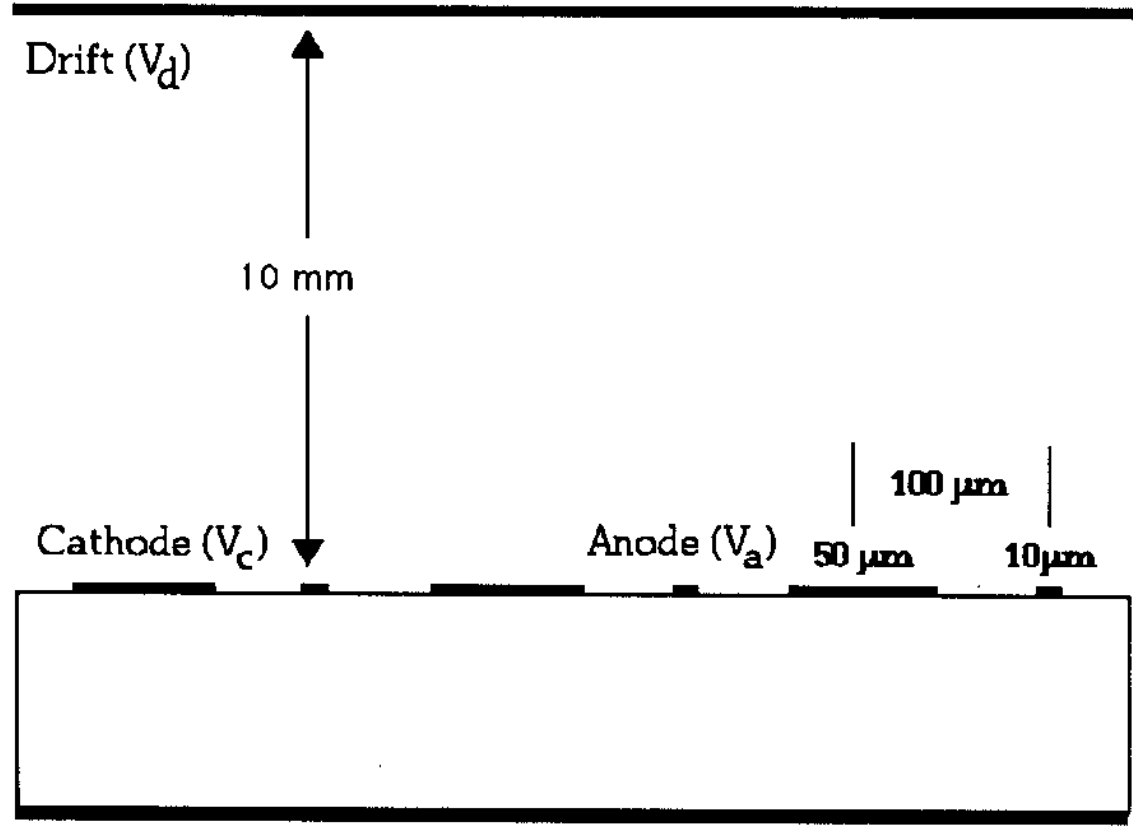

Back Plane $\left(V_{b}\right)$

Fig. 1

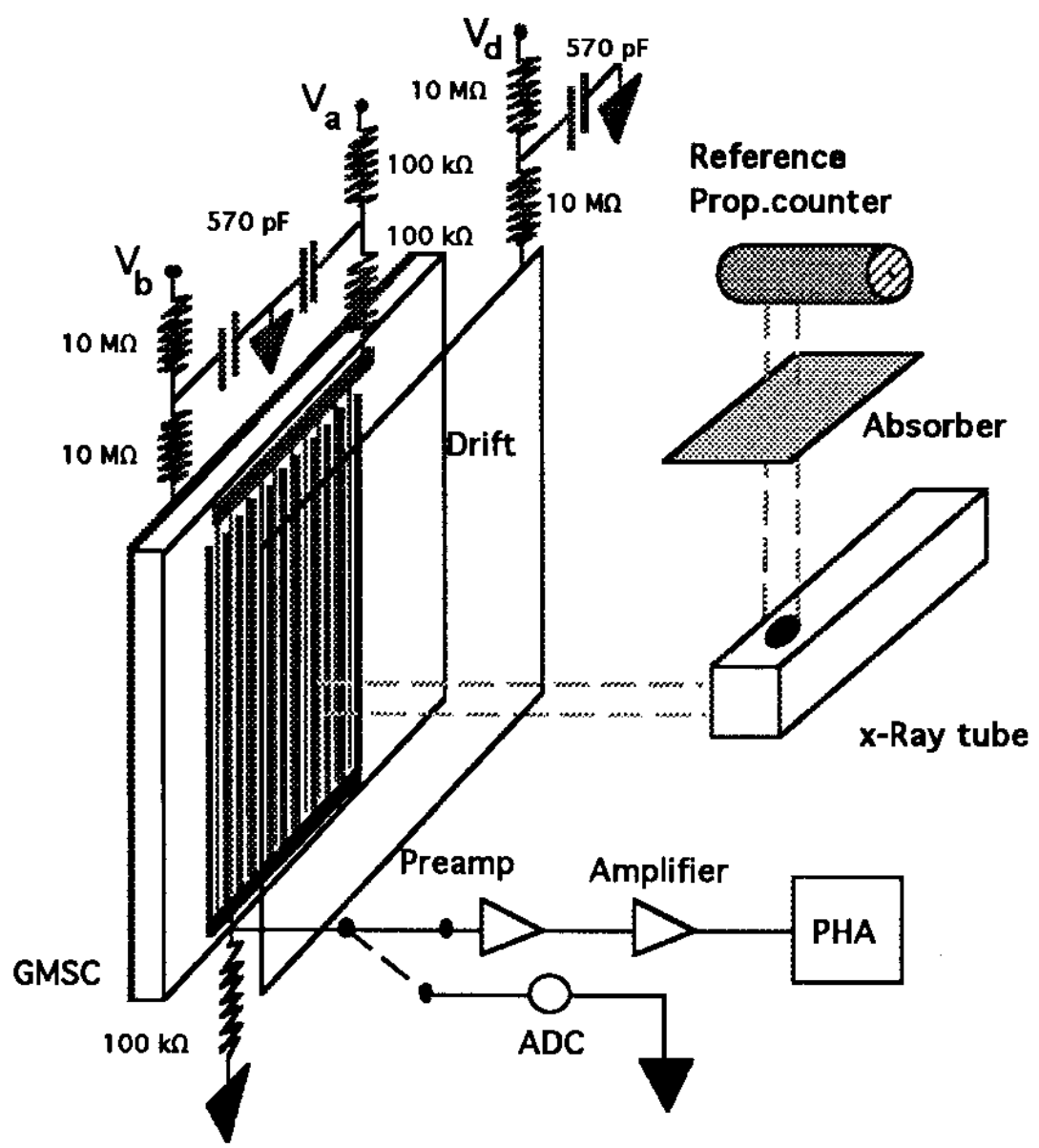

Fig. 2 




Fig. 3

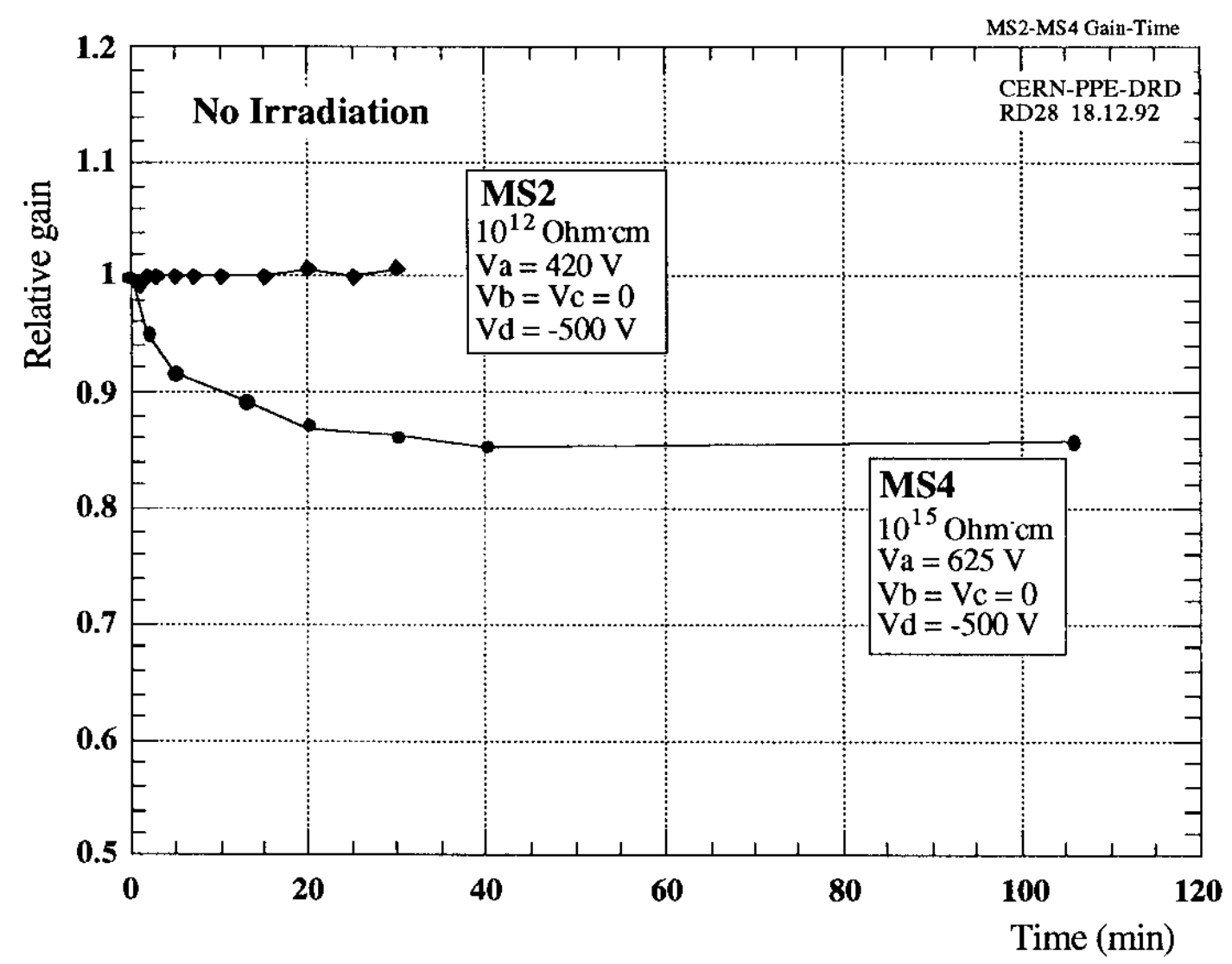

Fig. 4 


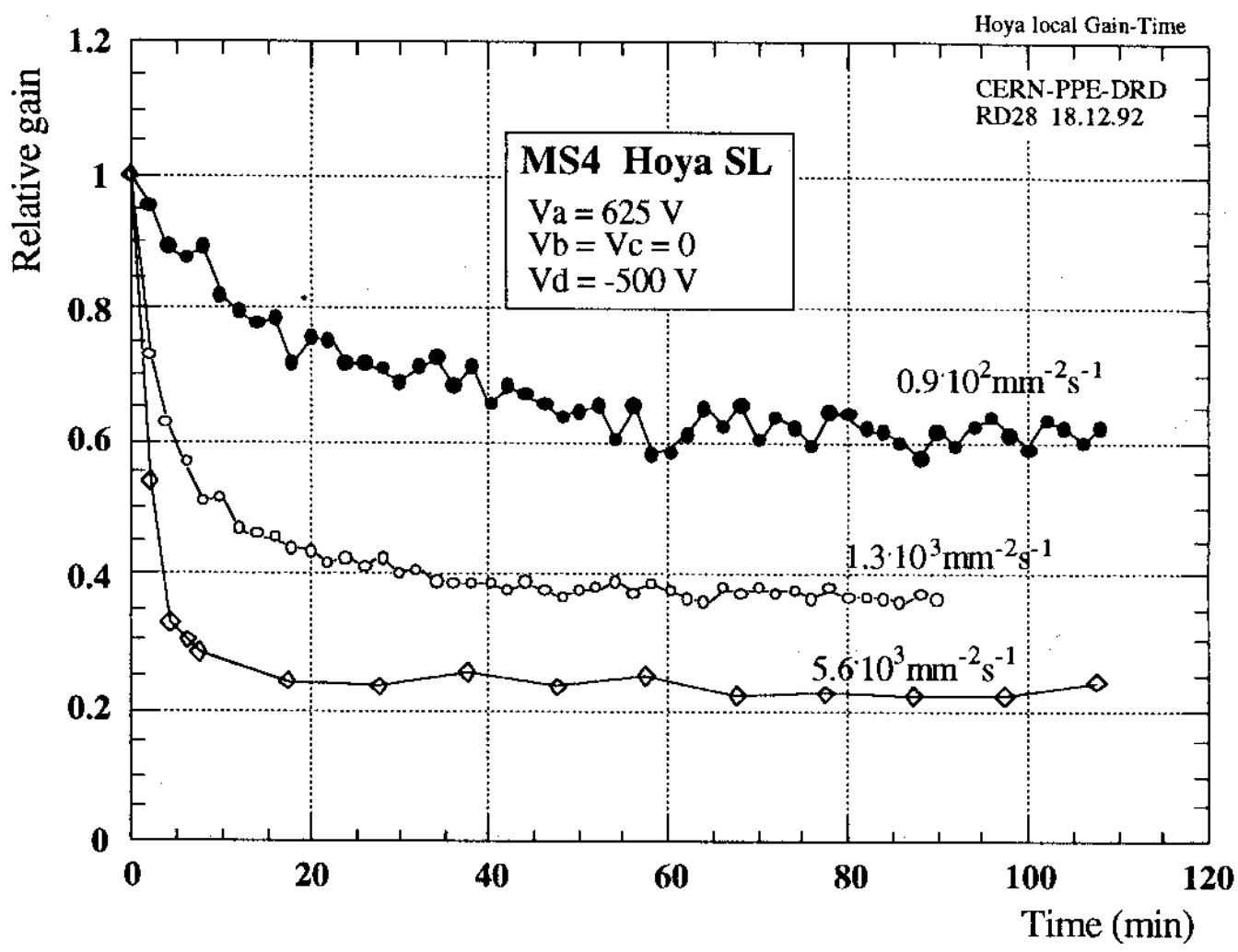

Fig. 5

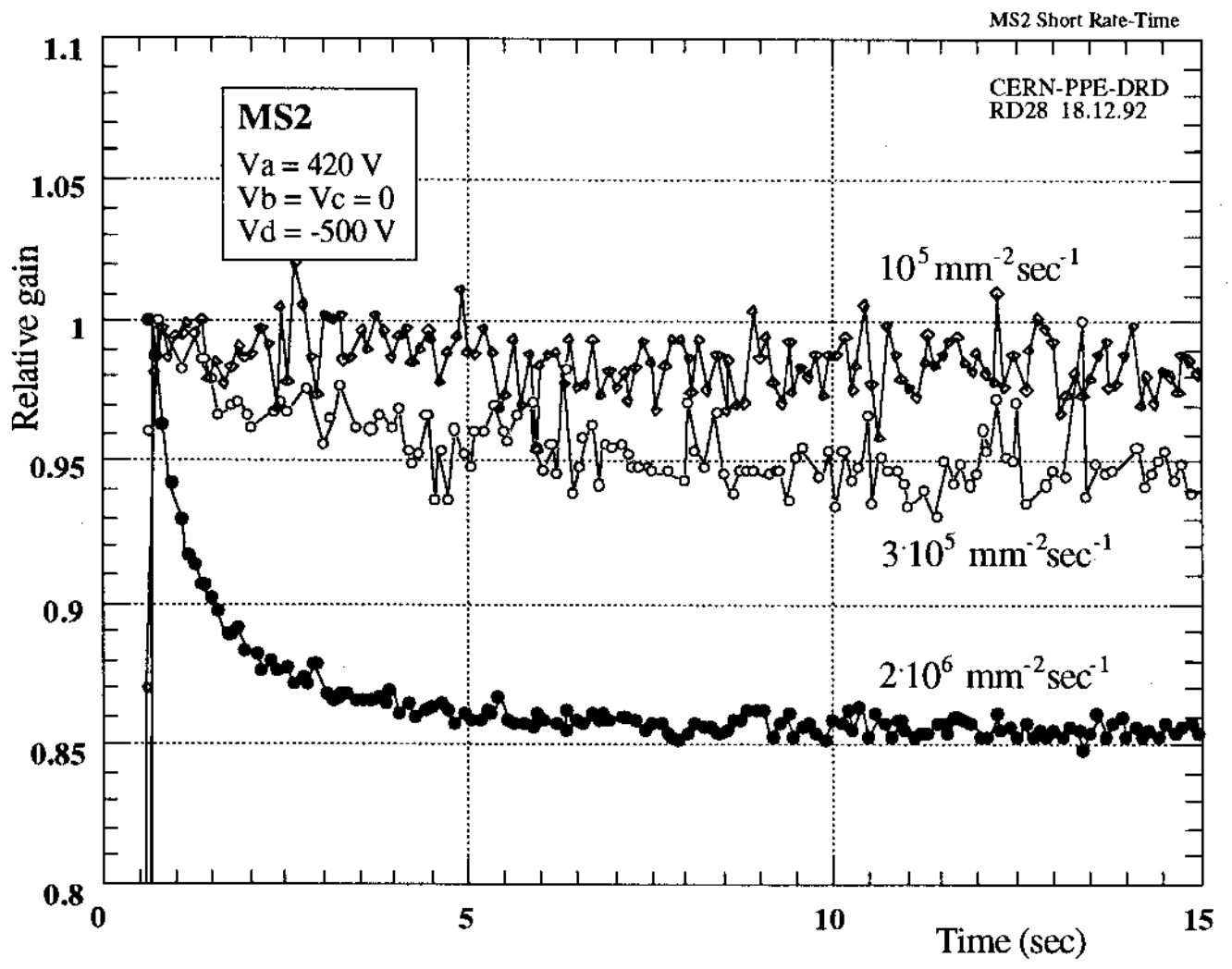

Fig. 6 


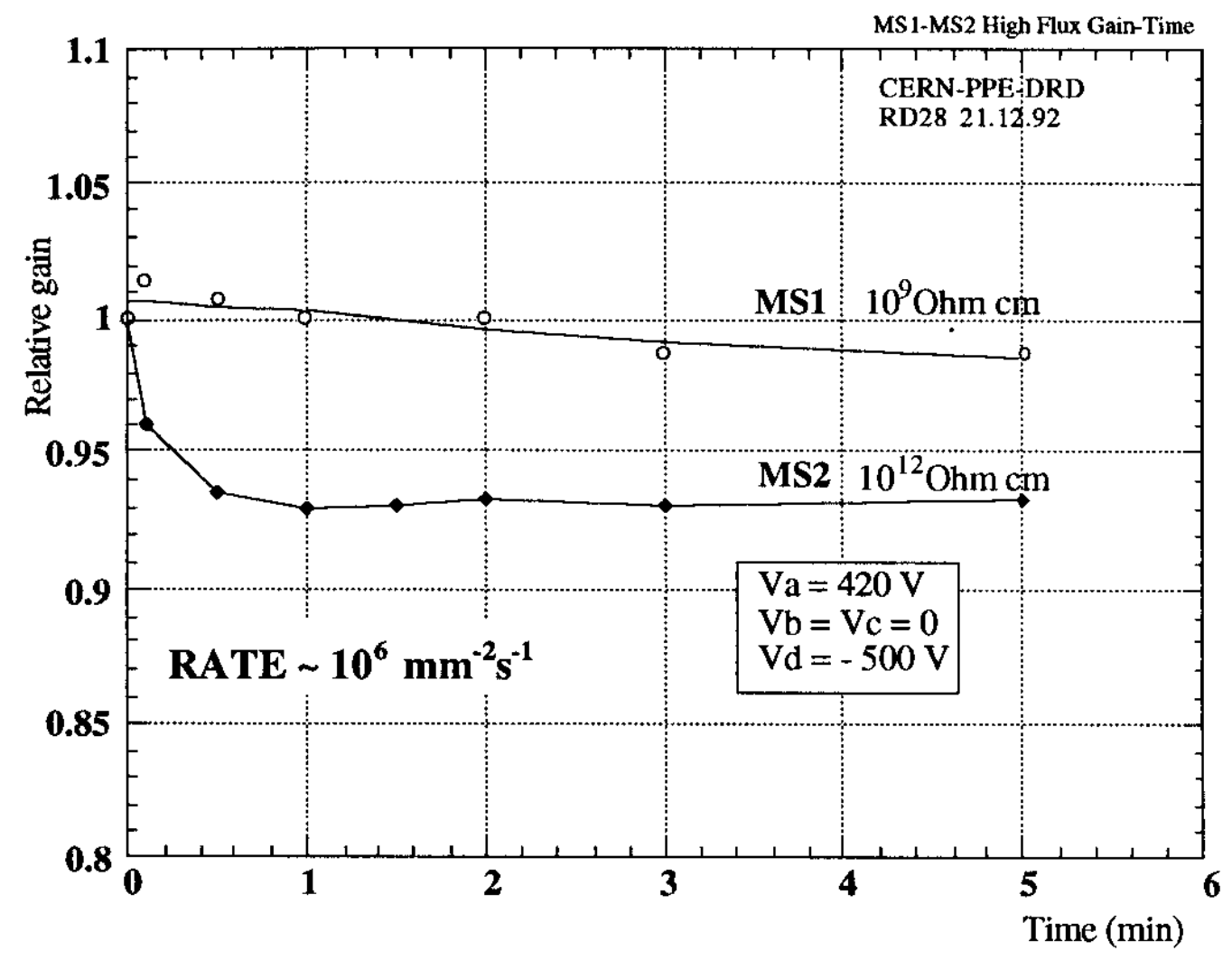

Fig. 7

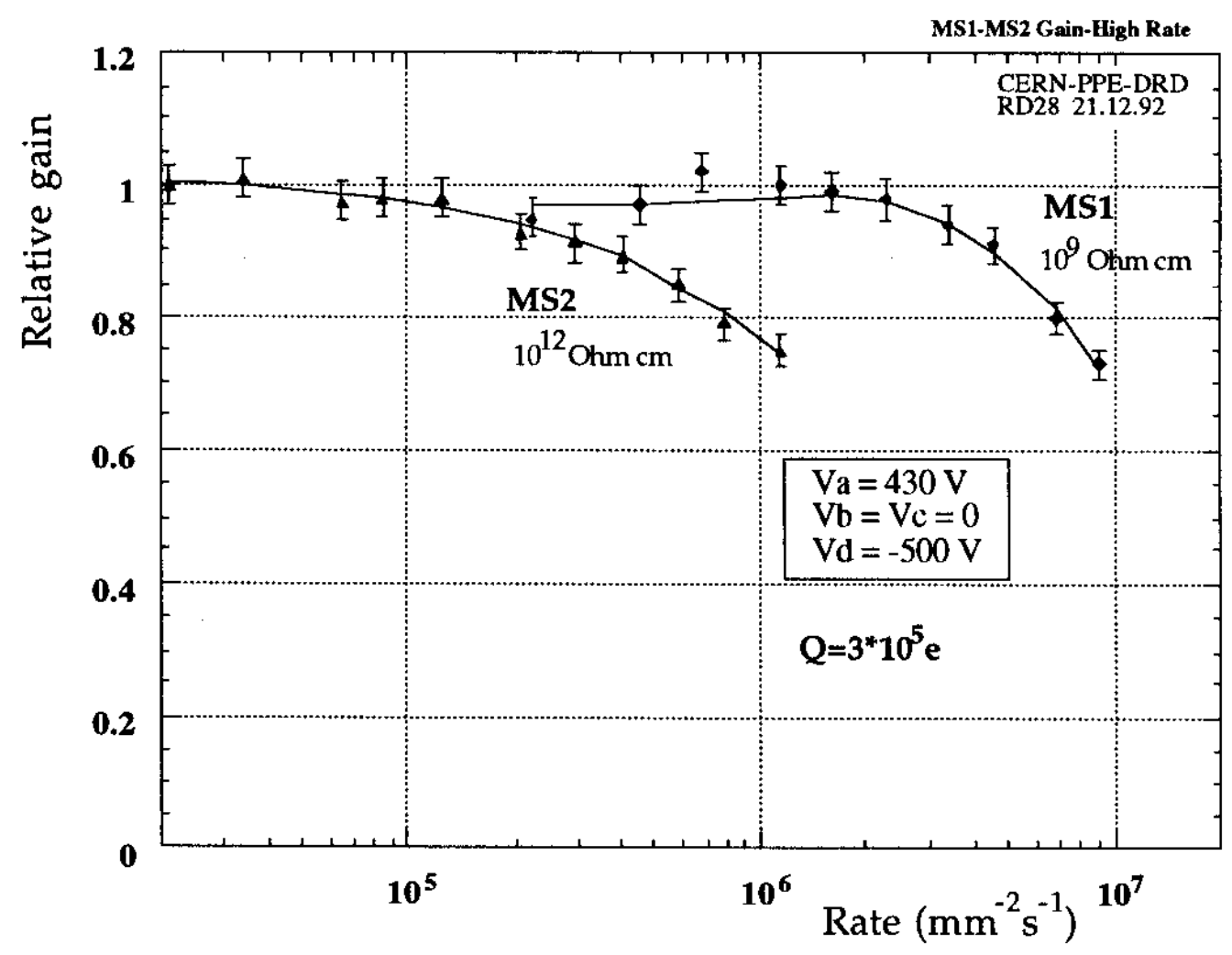

Fig. 8 


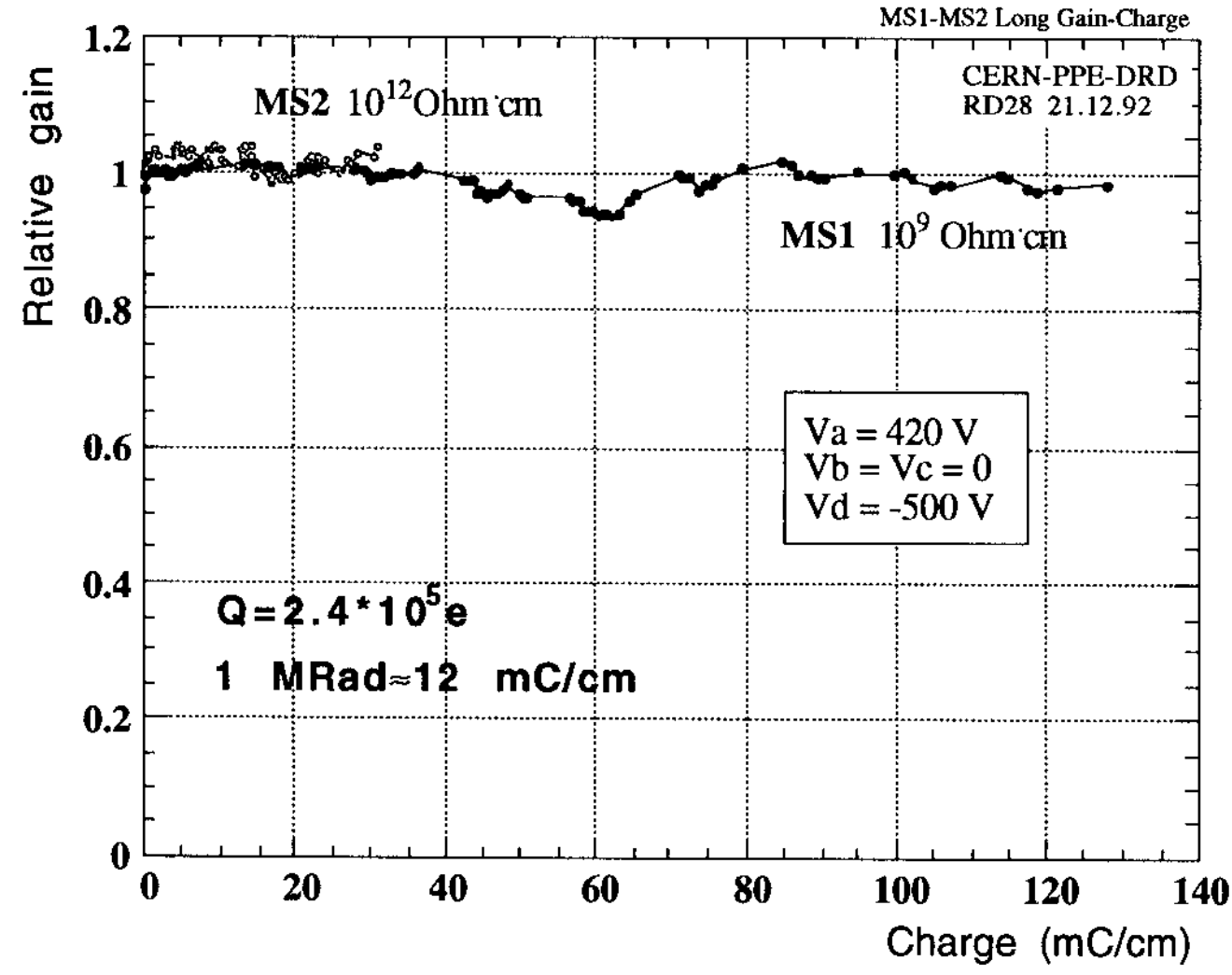

Fig. 9

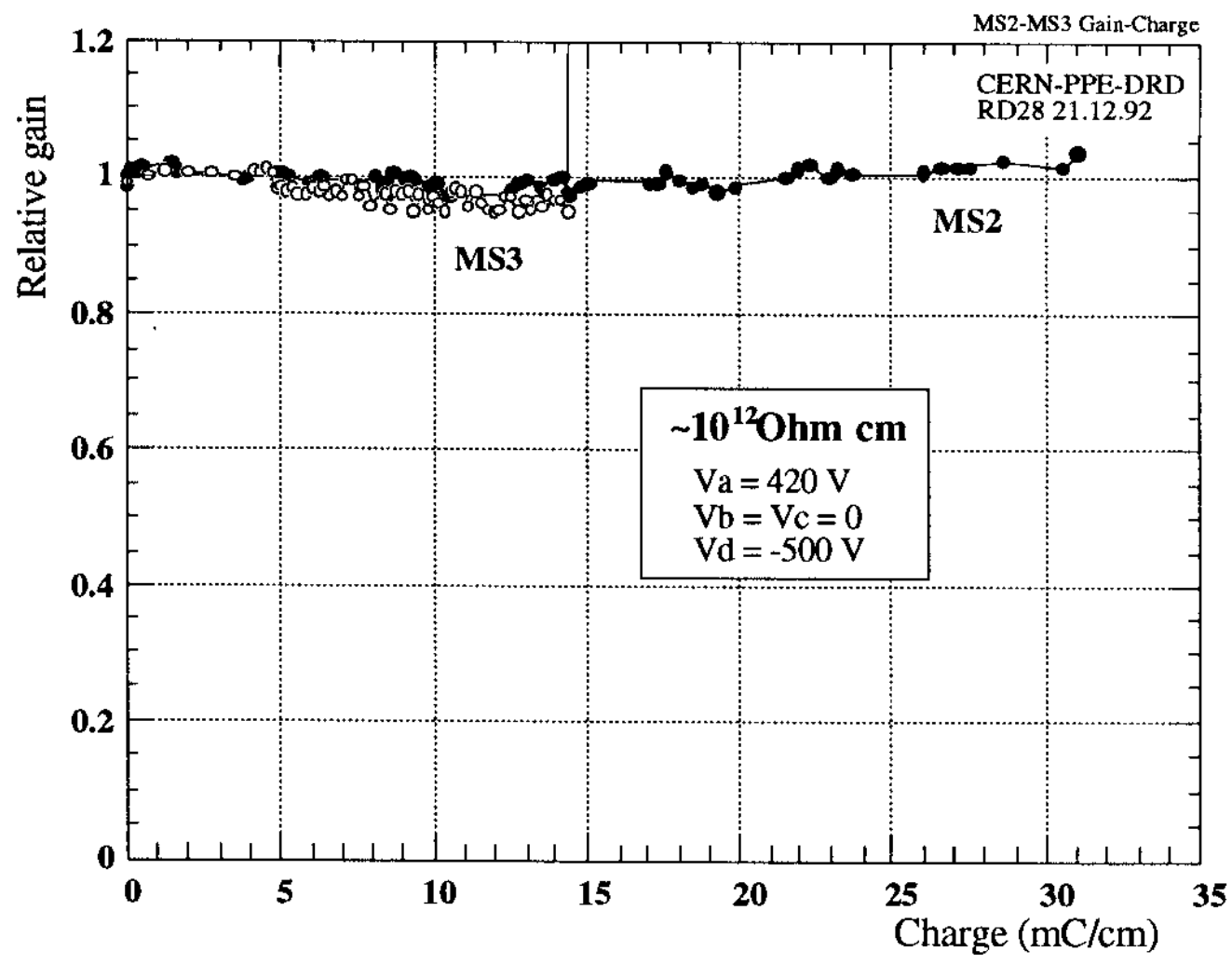

Fig. 10 